\title{
Repeated Dribbling Ability in Young Soccer Players: Reproducibility and Variation by the Competitive Level
}

\author{
by \\ João P. Duarte ${ }^{1}$ Óscar Tavares ${ }^{3}$, João Valente-dos-Santos ${ }^{1,2}$, Vitor Severino1, \\ Alexis Ahmed ${ }^{1}$, Ricardo Rebelo-Gonçalves ${ }^{1}$, João R. Pereira ${ }^{1}$, Vasco Vaz', \\ Susana Póvoas ${ }^{4}$, André Seabra ${ }^{5}$, Sean P. Cumming ${ }^{6}$, Manuel J Coelho-e-Silva ${ }^{1}$
}

The intermittent nature of match performance in youth soccer supports relevance of ability to repeatedly produce high-intensity actions with short recovery periods. This study was aimed to examine the reproducibility of a repeated dribbling ability protocol and, additionally, to estimate the contribution of concurrent tests to explain interindividual variability in repeated dribbling output. The total sample comprised 98 players who were assessed as two independent samples: 31 players were assessed twice to examine reliability of the protocol; and 67 juveniles aged $16.1 \pm$ 0.6 years were compared by the competitive level (local, $n=34$; national, $n=33$ ) to examine construct validity. All single measurements appeared to be reasonably reliable: total (ICC $=0.924 ; 95 \%$ CI: 0.841 to 0.963); ideal (ICC $=0.913$; 95\%CI: 0.820 to 0.958); worst (ICC $=0.813 ; 95 \%$ CI: 0.611 to 0.910). In addition, the percentage of the coefficient of variation was below the critical value of $5 \%$ for total $(\% C V=3.84$; $T E M=2.51 \mathrm{~s})$; ideal $(\% C V=3.90, T E M=2.48 \mathrm{~s})$. Comparisons between local and national players suggested magnitude effects as follows: moderate (d-value ranged from 0.63 to 0.89$)$ for all repeated sprint ability scores; large for total $(d=1.87)$, ideal $(d=1.72)$, worst $(d=1.28)$ and moderate for composite scores: the fatigue index $(d=0.69)$ and the decrement score $(d=0.67)$. In summary, the dribbling protocol presented reasonable reproducibility properties and output extracted from the protocol seemed to be independent from biological maturation.

Key words: short-term maximal effort; intra-class correlation; reliability.

\section{Introduction}

Soccer is the most popular sport in the world with 265 million registered participants worldwide (FIFA, 2012). In Portugal, recent statistics suggest that more than 154 thousand players within the national federation (Portuguese Institute of Statistic, 2013) represent about $29 \%$ of youth participating in organized and competitive sports. In Portugal youth soccer is organized into four age-groups: infantiles (under-13), initiates (under-15), juveniles (under-17) and juniors (under-19). Seniors compere at the professional (18 clubs in the top league, 24 clubs in the second league) and amateur level (III division nationwide; regional competitions: level 1-3). Most, but not all of the top soccer clubs competing at the professional level have youth academies.

On average, youth soccer players cover approximately $6 \mathrm{~km}$ during competitions, of which $16 \%$ of the distance is covered at speeds above $13.0 \mathrm{~km} \bullet \mathrm{h}-1$ (Castagna et al., 2009). Critical

\footnotetext{
1 - uid/dtp/03213/2016, Faculty of Sport Sciences and Physical Education, University of Coimbra, PORTUGAL.

2 - Faculty of Physical Education and Sport, ULHT, Lisbon, PORTUGAL.

3 - School of Health and Technology, Coimbra, PORTUGAL.

4 - CIDESD, University Institute of Maia (ISMAI), Maia, PORTUGAL.

5 - Faculty Sport, University of Porto, PORTUGAL.

6 - School for Health, University of Bath, UNITED KINGDOM.
} 
moments in soccer commonly consist of high intensity short-term efforts, either with or without the ball. The ability to sprint with the ball at high speed has been recognized as a desirable attribute in youth soccer (Malina et al., 2005; Reilly et al., 2000), as well as the ability of dribbling and/or rapidly changing directions and positions. The intermittent nature of match performance in soccer requires the ability to repeatedly produce high-intensity actions every $72 \mathrm{~s}$ (Bradley et al., 2009). Moreover, literature indicates that 1.2 to $2.4 \%$ of the total covered distance is performed with the ball (Di Salvo et al., 2007). Data from matches of the English FA Premier League, using the Prozone ${ }^{\circledR}$ path identification system, identified changes of directions between $45^{\circ}$ and $135^{\circ}$ as the most frequent, in comparison to directional changes of $<135^{\circ}$ (Robinson et al., 2011).

The relationships between repeated sprint ability and other performance attributes in soccer, such as acceleration, agility, explosive leg power and aerobic conditioning through the age groups (U11 to U18) were examined using a $6 \times 30 \mathrm{~m}$ sprint protocol. Despite some variation induced by age, it was found that repeated sprint ability correlated positively with other tests (Spencer et al., 2011), what is in accordance with the results of previous studies (Young et al., 1996). Although a positive relationship between sprinting and dribbling has been well documented, it should be noted that the skill of dribbling is complex and may be influenced by a number of additional factors. Indeed, previous research suggests that amateur and professional soccer players differ in developmental changes in dribbling (Huijgen et al., 2010). However, the extent to which developmental differences impact the relationship between sprinting and changes in directions is still unclear.

Coordinative performance tends to decrease as a result of fatigue (Cortis et al., 2011). Thus, coaches should include agility drills toward the end of a training session to better prepare players to cope with match demands. Within the multivariate domains of soccer, crucial attributes include the ability to control the ball with the feet, shoot at the goal with accuracy, dribble and retain possession. Tessitore et al. (2011) observed a decrease in performance of athletes in the first 10 days of the preseason, however, the best dribbling performances were obtained during the last training session. This suggests that despite temporary decreases in performance during the period of high volume/intensity conditioning, positive agility adaptations occur after three weeks of practice. Another research studied the relationship between straight sprinting ability and change of direction sprint tests which was examined among 157 male rugby players aged 819 years. It was concluded that performance in change of direction in sprint tests was positively correlated with age and the mastery level of players, while straight sprint test performance depended exclusively on age (Condello et al., 2013). Literature also indicates that repeated sprint ability differentiates between players who drop out of competitive soccer and those who continue and are promoted (Figueiredo et al., 2009). Repeated dribbling ability may also serve as a positive predictor of talent in youth soccer (Ali, 2011). It could be argued that it is a more ecologically valid test of ability as it requires the athlete to perform with the ball at their feet. A candidate test in talent identification should be able to discriminate players by competitive levels. Moreover, motor tests need to be characterized by reproducibility (Barrow et al., 1989). With this in mind, the present study aimed to examine the reproducibility of a repeated dribbling ability protocol, to estimate the contribution of concurrent protocols (sprint and dribbling) in the classification of young soccer players by the competitive level and, finally, identify the correlates of repeated dribbling output (total time, ideal time, a fatigue index and a decrement score).

\section{Material and Methods}

\section{Participants}

The sample included 98 soccer players with a mean age of $16.0 \pm 1.1$ years. Information regarding training experience and the playing position was obtained from each player and checked by club records. The study included two subsamples: a) 31 players were assessed twice to examine reliability of the RDA test; b) 67 juveniles were tested for comparisons considering the competitive level to examine construct validity (local: $\mathrm{n}=34$; national: $\mathrm{n}=33$ ).

\section{Procedures}

The Scientific Committee of the Faculty of Sports Sciences and Physical Education of the 
University of Coimbra approved the study. Subsequently, four clubs were contacted. Parents or legal guardians provided informed consent and players were informed that their participation was voluntary and that they could withdraw from the study at any time. All procedures were in accordance with ethical standards for sports medicine (Harriss and Atkinson, 2009). Tests were performed on the same weekday and at the same time period (i.e. 16:00-20:00) to avoid variation due to the circadian rhythm. Test conditions were controlled and test sessions were performed on artificial grass under similar conditions (Oregon Scientific RMR383HG: air temperature 24.8-30.1 ${ }^{\circ}$ C; humidity $30-41 \%$ ).

\section{Chronological age and maturation}

Chronological age (CA) was calculated during the first visit to the clubs. Regarding biological maturation, somatic maturation was assessed as the percentage of estimated mature stature (EMS\%). This non-invasive method was developed by an algorithm proposed by Khamis and Roche (1994). Predicted mature stature was derived from chronological age, stature, body mass and mid-parent stature. Afterwards, current stature was expressed as the percentage of predicted mature stature and for two players of the same age; the one who was closer to mature stature was more advanced in biological maturation than the individual who was farther from mature stature.

\section{Body size variables}

Body mass (BM) and stature were measured to the nearest $0.1 \mathrm{~kg}$ and $0.1 \mathrm{~cm}$ using a scale (SECA 770, Hanover, MD, USA) and a stadiometer (Harpenden 98.603, Holtain Ltd, Croswell, UK), respectively. The triceps and medial calf skinfolds were measured using a Lange Caliper (Beta Technology, Ann Arbor, MI, USA), and fat mass (FM) was estimated using equations from the Slaughter et al.'s study (1988). Fat-free mass (FFM) was also calculated. Anthropometric evaluation of total thigh volume (TV $\left.\mathrm{TNTH}_{\mathrm{AN}}\right)$ of the dominant leg was obtained from three circumferences and partial lengths. The thigh was partitioned into two segments which resembled truncated cones. Circumferences were measured at the most possible proximal circumference, the middle thigh and above the patella (minimum). The lengths between consecutive circumferences were also measured.
Calculation of the volume of each assumed truncated cone volume was based on the equation proposed by Jones and Pearson (1969). The original study considered six truncated segments of the leg (seven circumferences, six lengths). In the current study, TV ANTH was calculated as the sum of volumes derived from two truncated cones: truncated cone A was determined from circumferences measured at gluteal furrow and the mid-thigh; truncated cone B was similarly determined by using circumferences at the midthigh and at the transversal plane above the patella.

\section{Standing long jump and sit-ups}

As in previous studies (Coelho-e-Silva et al., 2012; Freitas et al., 2014), a standing long jump (SLJ) was used to test power and a sit-up test was used to evaluate the dynamic endurance of abdominal muscles. Both tests were performed according to Council of Europe (1988) guidelines. For the SLJ the subject stood just behind the takeoff line with the feet together and was instructed to jump as far as possible. The test was performed twice and the better score was used for further analysis. When testing sit-ups the subject lay on his back on the mat with the arms folded and the hands on the shoulders. The knees were bent $(90$ degrees) and the heels were in contact with the floor. The participants' ankles were held by another subject who counted aloud each time a complete sit-up was performed. With each sit-up the elbows had to touch the knees.

\section{Repeated sprint ability}

Repeated sprint ability (RSA): Short-term maximal output (RSA: 7 × $35 \mathrm{~m} / 25^{\prime \prime}$ ) was assessed as described by Bangsbo (1994). This test corresponds to seven consecutive $35 \mathrm{~m}$ sprints including changes of directions and a recovery period of $25 \mathrm{~s}$ between sprints during which the subject runs or walks from the end line back to the starting line. The time for each sprint was recorded by a digital chronometer connected to photoelectric cells (Globus Ergo Tester Pro). The following variables were determined: a) the fastest sprint, b) the slowest sprint, c) the sum of all sprints, d) the ideal time that corresponded to the best sprint multiplied by six. Two additional composite scores were considered: e) fatigue time: the worst sprint minus the best sprint (Bangsbo, 1994); and f) a decrement score (Bishop et al., 2001): 
100 * (sum 7 sprints / ideal time) -100 .

\section{Repeated dribbling ability}

Repeated dibbling ability (RDA-7): A modified version of RSA was also performed (RDA-7: 7x35-m/25"). This protocol resembles the one of RSA assessment as it consists of seven consecutive $35 \mathrm{~m}$ runs with changes of directions with the ball, including a $25 \mathrm{~s}$ recovery period between runs. The time for each sprint was recorded by a digital chronometer connected to photoelectric cells (Globus Ergo Tester Pro). The same RSA output was retained for subsequent analyses: a) the fastest sprint, b) the slowest sprint, c) the sum of all sprints, d) the ideal time that corresponded to the best sprint multiplied by seven. Two additional composite scores were considered: e) fatigue time; f) a decrement score. The standing long jump was used to test the power (Council of Europe, 1988). The test was performed twice and the better score was registered. A sit-up test was used to evaluate the dynamic endurance of abdominal muscles (Council of Europe, 1988). The test was performed once and the total number of correctly performed and fully completed sit-ups within the $30 \mathrm{~s}$ period was taken as the score.

\section{Analysis}

Descriptive statistics were reported (means \pm standard deviations) and standard error of the means for the total sample $(n=98)$. Additionally, based on a subsample $(\mathrm{n}=31)$, means and standard deviations at time moments 1 and 2 (one week apart) were reported. The intra-class correlation coefficient (ICC) was calculated in parallel to technical error of measurement (TEM) following the equation proposed by Mueller and Martorell (1988). The coefficient of variation (\%CV and respective $95 \%$ confidence intervals) was expressed as the percentage of the mean. Performance output included total time (sum of all trials), ideal time (best trial multiplied by the number of trials) and the worst trial. Comparisons between under-17 adolescent soccer players classified by the competitive level (local, $\mathrm{n}=34$; national, $n=33$ ) were performed using an independent $t$-test in addition to effect size evaluation. Cohen's d effect sizes (ES) and thresholds $(<0.2,0.2-0.6,0.6-1.2,1.2-2.0,2.0-4.0$ for trivial, small, moderate, large, very large and extremely large, respectively) were also used to compare the magnitude of the differences in physical and technical performance (Hopkins, 2004). The smallest worthwhile difference was set at Cohen's effect size of 0.2 as recommended for team sports and fitness tests (Ali, 2011; Hopkins, 2000). Discriminant function analysis was subsequently performed to obtain the best predictor to classify under-17 soccer players into their original competitive groups. Finally, multiple linear regression analyses (backward method) were used to obtain predictive models for each of the RDA output from a pool of candidate determinants (training experience, chronological age, somatic maturation, stature, body mass, \% fat mass, estimated thigh volume, sit-ups and a standing long jump). All statistical analyses were performed using IBM SPSS v.20 for Mac OS software (SPSS Inc., IBM Company, NY, USA).

\section{Results}

Descriptive statistics for adolescent soccer players aged $16.0 \pm 1.1$ years $(n=98)$ are presented in Table 1 which includes training experience, decimal age, biological maturation given as the attained percentage of predicted mature stature, anthropometry and performance scores derived from the two protocols: repeated sprint ability and repeated dribbling ability. Training experience ranged from 2 to 12 years. Thirteen players $(13.3 \%)$ were already mature since they attained $100 \%$ of the predicted mature stature. The analysis of reproducibility (Table 2) was conducted for a subsample of 31 soccer players (under-15: $n=10$; under-17: $n=11$; under-19: $n=10$ ) extracted from the total sample presented in Table 1. From session 1 to session 2, eleven players improved their performance in RDA-total, fourteen improved their RDA-ideal scores and fifteen obtained better scores in the RDA-worst output. All single measurements appeared to be reasonably reliable: RDA-total $(\mathrm{ICC}=0.924$; 95\%CI: 0.841 to 0.963 ); RDA-ideal (ICC $=0.913$; $95 \%$ CI: 0.820 to 0.958 ); RDA-worst (ICC $=0.813$; 95\%CI: 0.611 to 0.910). Additionally, the percentage of the coefficient of variation was below the critical value of $5 \%$ for RDA-total (\%CV $=3.84 ; \mathrm{TEM}=2.51 \mathrm{~s})$; RDA-ideal $(\% \mathrm{CV}=3.90$, $\mathrm{TEM}=2.48 \mathrm{~s}$ ). 
Table 1

Descriptive statistics for the total sample $(n=98)$

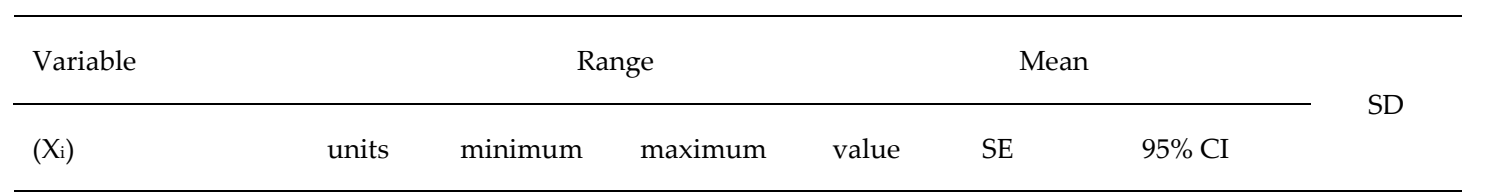

$\begin{array}{lccccccc}\text { Training experience } & \text { years } & 2.0 & 12.0 & 7.5 & 0.3 & \text { (6.99 to 8.03) } & 2.6 \\ \text { Chronological age } & \text { years } & 12.9 & 18.6 & 16.0 & 0.1 & \text { (15.8 to } 16.3) & 1.1 \\ \text { Attained EMS } & \% & 84.6 & 100.0 & 97.8 & 0.3 & \text { (97.2 to } 98.8) & 2.8\end{array}$

$\begin{array}{llllllll}\text { Stature } & \mathrm{cm} & 152.2 & 189.1 & 172.5 & 0.7 & \text { (171.1 to 174.0) } & 7.6 \\ & & & & & & & \end{array}$

Fat mass 11.6

$\begin{array}{llllllll}\text { Fat mass } & \mathrm{kg} & 4.9 & 28.2 & 10.9 & 0.5 & \text { (10.0 to } 11.9) & 5.0\end{array}$

$\begin{array}{llllllll}\text { Fat free mass } & \mathrm{kg} & 31.4 & 77.7 & 53.6 & 0.8 & \text { (52.0 to } 55.3) & 8.4\end{array}$

\section{EMS}

Thigh volume

L $\quad 3.19$

11.82

5.26

0.18

$(4.93$ to 5.65$) \quad 1.74$

RSA total time

$\mathrm{s}$

46.58

71.80

51.60

0.35

(50.90 to 52.29)

3.47

RSA ideal time

42.56

64.47

$49.53 \quad 0.30$

(48.94 to 50.12$)$

2.94

RSA worst trial

6.81

10.96

$\begin{array}{ll}7.71 & 0.06\end{array}$

(7.58 to 7.83 )

0.61

RDA total time

$\mathrm{s}$

54.17

85.34

63.80

0.57

(62.67 to 64.92$)$

5.62

RDA ideal time

50.89

79.76

60.30

0.48

(59.35 to 61.24$)$

4.71

RDA worst trial

8.03

15.56

9.90

$0.14 \quad$ (9.62 to 10.18)

1.38

(estimate mature stature); RSA (repeated sprint ability);

RDA (repeated dribbling ability); SE (standard error);

95\% CI (95\% confidence interval); SD (standard deviation) 


\section{Table 2}

Correlations between sessions, technical error of measurements (TEM), coefficient of variation $(\% \mathrm{CV})$ and intra-class correlation coefficient (ICC) with respective $95 \%$ CI $(n=31)$

\begin{tabular}{lcccccc}
\hline \multirow{2}{*}{ Variables } & $\begin{array}{c}\text { Time } \\
\text { moment 1 }\end{array}$ & $\begin{array}{c}\text { Time } \\
\text { moment 2 }\end{array}$ & & \multirow{2}{*}{ TEM } & \%CV \\
\cline { 2 - 5 } & Mean \pm SD & Mean \pm SD & Value & (95\% CI) & & \\
\hline & & & & & & \\
RDA total time, s & $67.20 \pm 6.17$ & $68.55 \pm 6.87$ & 0.924 & $(0.841$ to 0.963$)$ & 2.61 & 3.84 \\
RDA ideal time, s & $63.15 \pm 5.67$ & $63.98 \pm 6.57$ & 0.913 & $(0.820$ to 0.958$)$ & 2.48 & 3.90 \\
RDA worst trial, s & $10.51 \pm 1.56$ & $10.67 \pm 1.29$ & 0.813 & $(0.611$ to 0.910$)$ & 0.80 & 7.53
\end{tabular}

RDA (repeated dribbling ability); TEM (technical error of measurements);

$\% C V(\%$ coefficient of variation); ICC (intra-class correlation coefficient);

95\% CI (95\% confidence interval); RDA (repeated dribbling ability);

$R D A$ total time (sum of the seven trials); RDA ideal time (corresponds

to the best trial multiplied by seven); RDA worst trial

(corresponds to the poorest performance in the repeated trials)

Table 3

Descriptive statistics for performance output (sit-ups, standing long jump, $R S A$ and RDA) by the competitive level and comparison between groups $(n=67)$

Yi:

Dependent variables

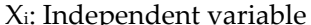
Mean \pm SD (95\% CI of mean)

Local $(\mathrm{n}=34) \quad$ National $(\mathrm{n}=33)$
Difference between groups Effect size: (95\%CI of mean) d (qualitative)

$\begin{array}{lccccr}\text { Sit-ups, rep } & 29 \pm 4 & 31 \pm 3 & +2(+1 \text { to }+4) & 0.65 \text { (moderate) } & 0.13 \\ \text { SLJ, cm } & 209 \pm 14 & 221 \pm 14 & +12(+6 \text { to }+19) & 0.88 \text { (moderate) } & 0.18 \\ \text { RSA-total, s } & 51.58 \pm 2.65 & 49.71 \pm 1.64 & +1.861(+0.782 \text { to }+2.940) & 0.83 \text { (moderate) } & 0.37 \\ \text { RSA-ideal, s } & 49.47 \pm 2.38 & 48.20 \pm 1.46 & +1.265(+0.297 \text { to }+2.233) & 0.63 \text { (moderate) } & 0.25 \\ \text { RSA-worst, s } & 7.74 \pm 0.53 & 7.35 \pm 0.30 & +0.395(+0.183 \text { to } 0.606) & 0.89 \text { (moderate) } & 0.08 \\ \text { RSA-FI, s } & 0.68 \pm 0.42 & 0.46 \pm 0.20 & +0.214(+0.053 \text { to }+0.375) & 0.66 \text { (moderate) } & 0.04 \\ \text { RSA-DS, \% } & 4.28 \pm 2.53 & 3.14 \pm 1.26 & +1.140(+0.161 \text { to }+2.118) & 0.86 \text { (moderate) } & 0.35 \\ \text { RDA-total, s } & 65.34 \pm 4.17 & 59.01 \pm 2.16 & +6.326(+4.696 \text { to }+7.951) & 1.87 \text { (large) } & 1.27 \\ \text { RDA-ideal, s } & 61.27 \pm 2.59 & 56.62 \pm 2.32 & +4.647(+3.351 \text { to }+5.943) & 1.72 \text { (large) } & 0.93 \\ \text { RDA-worst, s } & 10.26 \pm 1.33 & 8.95 \pm 0.49 & +1.312(+0.821 \text { to }+1.803 & 1.28 \text { (large) } & 0.26 \\ \text { RDA-FI, s } & 1.51 \pm 1.22 & 0.86 \pm 0.46 & +0.649(+0.196 \text { to }+1.102) & 0.69 \text { (moderate) } & 0.13 \\ \text { RDA-DS, \% } & 6.65 \pm 4.59 & 4.25 \pm 1.80 & +2.395(+0.683 \text { to }+4.106) & 0.67 \text { (moderate) } & 0.48\end{array}$

SLJ (Standing long jump); RSA (repeated sprint ability); RDA (repeated dribbling ability); FI (fatigue index); DS (decrement score); SD (standard deviation); d (effect size value); SWD (smallest worthwhile difference) 


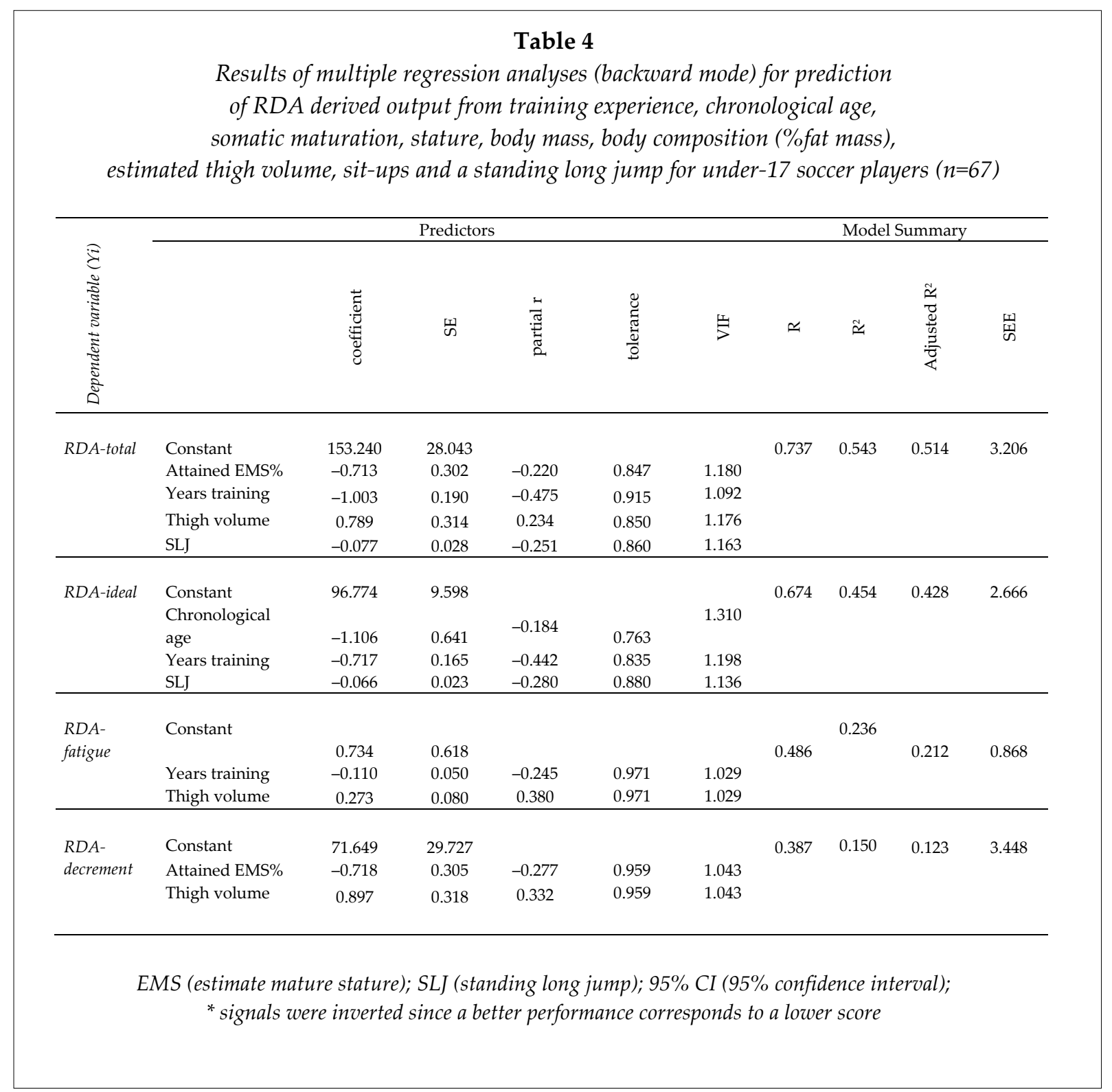

In contrast, the worst dribbling trial presented a TEM of $0.80 \mathrm{~s}(\% \mathrm{CV}=7.53)$.

The comparison of the under-17 players classified as local and national is presented in Table 3. It is possible to note an overall trend towards better performance in the national group on output derived from both the RSA and RDA protocols. The magnitude of these effects was moderate (d-value ranged from 0.63 to 0.89 ) for all RSA scores while for RDA effect size was large for RSA-total ( $\mathrm{d}=1.87)$, RDA-ideal $(\mathrm{d}=1.72)$, RDAworst $(\mathrm{d}=1.28)$ and moderate for composite scores: the fatigue index $(d=0.69)$ and decrement score $(d=0.67)$. Furthermore, it was possible to perform a significant discriminant function analysis (stepwise method: Wilks' Lambda = 0.520, $\left.\mathrm{df}_{1}=1, \mathrm{df}_{2}=1, \mathrm{df}_{3}=65 ; \mathrm{r}_{\mathrm{c}}=0.693\right)$ that correctly classified $58(86.6 \%)$ from 67 under- 17 soccer players into the original groups. The model had a single significant predictor: RDA-total $(\mathrm{F}=$ 60.116, $\left.\mathrm{df}_{1}=1, \mathrm{df}_{2}=65, p<0.01\right)$.

Table 4 presents the relations among training experience, chronological age, somatic maturation, anthropometry and output derived from the RDA test. Backward multiple linear regression analysis suggested that $51.4 \%$ of the inter-individual variance in RDA-total time was explained by four predictors. Explained variance 
for RDA-ideal time was $42.8 \%$ and based on three predictors. Fatigue index and decrement scores presented $21.2 \%$ and $12.3 \%$, respectively, of explained variance from models that uniquely considered two independent variables. Training experience entered three times in the predictive models and seemed to be the most relevant determinant of RDA output. Lower limb explosive strength given by the standing long jump was a significant predictor for RDA-total and RDA-ideal times, but it did not enter in the composite scores (fatigue index and decrement score). Thigh volume emerged as a predictor of RDA-total and RDA-ds (decrement score) suggesting that larger volume corresponded to poorer performance in the mentioned output. Finally, somatic maturation was a significant determinant for the RDA-total time and RDAdecrement score.

\section{Discussion}

The current study examined the reliability and construct validity of the Repeated Dribbling Ability test as a protocol to assess soccer specific skills. The RDA test demonstrated acceptable reliability among young soccer players. Furthermore, variation of RDA output was found to be related to the competitive level (national level versus local level) as elite soccer players performed better than their local peers. Test retest reliability was classified as poor $(<0.40)$, fair (between 0.40 and $<0.70$ ), good (between 0.70 and $<0.90)$ and excellent $(\geq 0.90)$ following the guidelines suggested by Hopkins (2004). By inference, the RDA protocol produced excellent ICC values: 0.92 and 0.91 for total and ideal times, respectively. The current study also suggested significant relationships between repeated dribbling ability and training experience, age, somatic maturation, thigh volume as well as jumping performance. Finally, the magnitude of the mean difference between soccer players contrasting in the competitive level (local vs. national) was larger on two dependent variables: total time and ideal time (both from the repeated dribbling protocol).

Sprinting and running are fundamental movement skills for most sporting activities and are common forms of locomotion performed by children during playground games and activities. Furthermore, sprinting performance appears to be an important determinant of success in youth sports (Mendez-Villanueva et al., 2011). Assessments of maximal speed are commonly included in batteries of talent identification tests and studies focused on sport selection in youth soccer (Coelho-e-Silva et al., 2010). A significant change in leg length, standing height and mass across all maturation groups was reported in a previous study analyzing sprint performance and maturation in 332 boys aged 11-15 years (Meyers et al., 2015). Such growth-related changes are expected as part of natural processes of growth and maturation (Malina et al., 2004). Furthermore, stature and leg length were significantly related to speed, only when maturation was not controlled for. This suggests that the relationship between stature, leg length and speed is partially due to the influence of maturation in the sample population. Relationships between motor performance, as measured by various fitness tests, and age at peak height velocity were studied in a sample of 173 Flemish boys who were first measured yearly between 13 and 18 years and then again, as adults at 30 years of age (Lefevre et al., 1990). Comparisons were made between boys with an early, average and late age at peak height velocity and it was concluded that during adolescent years, three performance tasks, namely speed of limb movement, explosive strength and static strength, were negatively related to age at peak height velocity; thus, early maturers performed significantly better than late maturers. Maturity associated differences in most of these performance attributes are, however, generally attenuated or reversed in adulthood (Lefevre et al., 1990).

Variation in body size is also associated with contrasting maturity status in youth soccer players (Figueiredo et al., 2009), following patterns observed in the general population (Lefevre et al., 1990). Boys advanced in skeletal maturity are taller and heavier and possess greater absolute and relative lean mass than those on time and late in skeletal maturity (Malina et al., 2004). The differences among boys of contrasting maturity status also tend to persist when maturity-related variation in body height and mass is statistically controlled (Beunen et al., 1981). Performance differences among maturity groups were apparent by 13 years of age and tended to be greater at 14 and 15 years (Malina et 
al., 2004). The proportion of late maturing boys in elite adolescent soccer players also decreases with the competitive level and increasing chronological age (Malina et al., 2000), likely as a result of both the systematic and self-selection. Among adolescent soccer players performance tests showed a significant gradient of early $>$ on time>late among boys aged 13-14 years (Figueiredo et al., 2009). The impact of experience, body size and maturity status to variation in the functional capacities of adolescent soccer players was estimated in a sample of 69 players (aged 13.2-15.1 years) from Portuguese clubs competing in the highest division for their age; it was concluded that training was significantly related to aerobic resistance, whereas body size (body mass and stature) was a significant variable considering sprint and vertical jump tests (Malina et al., 2004).

Comparisons of the characteristics of regionally selected (regional elite, $\mathrm{n}=45$ ) and nonselected (local level, $\mathrm{n}=69$ ) under-14 players have considered body mass, stature, skinfolds, functional capacities, soccer-specific skills and goal orientation in addition to skeletal age assessed with the Fels method (Coelho-e-Silva et al., 2010). Selected players tend to present advanced maturity status, are heavier and taller; perform better in explosive power, repeated sprint ability and ball control; and are also more ego-oriented. The current study also examined variation by the competitive level in repeated sprint ability in parallel to assessment in repeated dribbling ability and differences were larger in RDA output as presented in Table 3. Of note, the discriminant function that was obtained to classify players by the competitive level retained RDA output (total time) as crucial information in parallel to years of practice, chronological age and fat free mass.

Predictors of functional capacities and skills in 143 soccer players aged 11-14 years from five clubs in Portugal were investigated using multiple linear regression analysis (Figueiredo et al, 2011). The explained variances differed among the various functional capacities (22-48\%) and skills (25\%). Skeletal maturity was a predictor of the countermovement jump. For repeated sprints, training history (positive), adiposity (negative) and body mass (negative) accounted for $27 \%$ of the variance in the fastest sprint. In the current study RDA-total was related to lower limb strength given by jumping performance, thigh volume, years of practice and the attained percentage of estimated mature stature. The other output derived from RDA protocols appeared independent of maturation, which may be very important for talent identification. Circumpubertal Flemish soccer players were successfully classified by the competitive level based on tests such as a $30 \mathrm{~m}$ dash, a shuttle sprint, an endurance shuttle run, sit-ups, sit-andreach combined with manipulative motor skills such as lobbing, juggling and dribbling (Vaeyens et al., 2006). Another independent study (Figueredo et al., 2009) compared the baseline physical growth and biological maturity status, functional capacities, sport-specific skills and goal orientation of youth soccer players who subsequently discontinued participation in the sport (drop-out), continued to participate at the same level (club) or moved to a higher playing standard (elite). Elite players performed better than dropouts in ball control, dribbling speed and the wall pass, and when compared to club players they were only better in the wall pass. Club players performed better than dropouts in dribbling speed and the wall pass.

In the present study, the repeated dribbling trial presented the lowest reliability coefficient (0.81) among the variables of interest. The data of the current study presented ideal and total time having larger coefficients (0.91-0.93). Similar results were found in recent soccer studies applying the running anaerobic sprint test (RAST) with a small sample $(n=14)$ of under-17 soccer players (de Andrade et al., 2014), where a mean time ICC of 0.96 was obtained. Another study noted that performance in kicking velocity among soccer players aged 12-15 years old revealed an ICC of 0.87 (Berjan-Bacvarevic et al., 2012). For the test of changes of direction, Lockie et al. (2013) determined an ICC value of 0.86 for the total time performance. Future studies may consider conducting 7 trials retaining the best 6 , with respect to the Bangsbo fatigue index (worst minus best). Recommendations for effective warm-up routines vary depending on the nature and duration of the exercise to be performed (Bishop, 2003a). Previous studies have shown importance of the warm-up and preceding activity performance, which consists of active or passive 
warming of body tissues in preparation for physical or sport activity (Bishop, 2003b); in the present study, although the warm-up was monitored, the daily routines of the sample were not controlled. Warm-up routines should include, in general, whole-body movements for 5 to $10 \mathrm{~min}$ followed by $5 \mathrm{~min}$ of recovery (Bishop, 2003b). The American College of Sports Medicine recommends a warm up including 5 to $10 \mathrm{~min}$ of callisthenic-type exercises and 5 to $10 \mathrm{~min}$ of progressive aerobic activity (ACSM, 2009). Regarding the decrement score, in addition to the potential erroneous fluctuations of the worst performance affecting the overall total time instead of the ideal time, there are some criticisms about using ratios between variables. Finally, the output obtained from the RDA protocol may probably need to consider qualitative information (the number of steps and ball touches in each trial) hypothesizing fatigue in movement inherent to the protocol.

\section{Acknowledgements}

In memorium of Filipe Simões, a beloved friend and colleague. This research was partially supported by Fundação para a Ciência e a Tecnologia [SFRH/BD/101083/2014; SFRH/BPD/100470/2014; uid/dtp/04213/2016]. The authors have no potential conflicts of interest.

\section{References}

Ali A. Measuring soccer skill performance: a review. Scand J Med Sci Sports, 2011; 21(2): 170-183

American College of Sports Medicine. ACSM's Guidelines for Exercise Testing and Prescription, 8th ed, Philadelphia: Lippincott Williams and Wilkins, 90; 2009

Bangsbo J. Fitness training in football - a scientific approach. Bagsvaerd: HO and Storm, 88-97; 1994

Barrow HM, Mckee R, Tritschler T. A Practical approach to measurement in physical education. Dubuque: WmC, Brown Publishers, 80-125; 1989

Berjan-Bacvarevic B, Pazin N, Bozic PR, Mirkov D, Kukolj M, Jaric S. Evaluation of a composite test of kicking performance. J Strength Cond Res, 2012; 26(7): 1945-1952

Beunen G, Ostyn M, Simons J, Renson R, Van Gerven D. Chronological and biological age as related to physical fitness in boys 12 to 19 years. Ann Hum Biol, 1981; 8: 321-331

Bishop D, Spencer M, Duffield R, Lawrence S. The validity of a repeated sprint ability test. J Sci Med Sport, 2001; 4(1): 19-29

Bishop D. Warm up I: potential mechanisms and the effects of passive warm up on exercise performance. Sports Med, 2003a; 33(6): 439-454

Bishop D. Warm up II: performance changes following active warm up and how to structure the warm up. Sports Med, 2003b; 33(7): 483-498

Bradley P, Sheldon W, Wooster B, Olsen P, Boanas P, Krustrup P. High-intensity running in English FA Premier League soccer matches. J Sport Sci, 2009; 27(2): 159-168

Castagna C, Impellizzeri F, Cecchini E, Rampinini E, Alvarez JC. Effects of intermittent-endurance fitness on match performance in young male soccer players. J Strength Cond Res, 2009; 23: 1954-1959 
Coelho-e-Silva MJ, Figueiredo AJ, Simões F, Seabra A, Natal A, Vaeyens R, Philippaerts R, Cumming SP, Malina RM. Discrimination of u-14 soccer players by level and position. Int J Sports Med, 2010; 31(11): 790-796

Coelho-e-Silva MJ, Vaz V, Simões F, Carvalho HM, Valente-Dos-Santos J, Figueiredo AJ, Pereira V, Vaeyens R, Philippaerts R, Elferink-Gemser MT, Malina RM. Sport selection in under-17 male roller hockey. J Sports Sci, 2012; 30(16): 1793-1802

Council of Europe. Eurofit: Handbook for the Eurofit tests of Physical Fitness. Rome: Council of Europe; 1988

Condello G, Minganti C, Lupo C, Benvenuti C, Pacini D, Tessitore A. Evaluation of change-of-direction movements in young rugby players. Int J Sports Physiol Perform, 2013; 8(1): 52-56

Cortis C, Tessitore A, Lupo C, Pesce C, Fossile E, Figura F, Capranica L. Inter-limb coordination, strength, jump, and sprint performances following a youth men's basketball game. J Strength Cond Res, 2011; 25(1): 135-142

de Andrade VL, Pereira Santiago PR, Kalva Filho CA, Campos EZ, Papoti M. Reproducibility of running anaerobic sprint test (rast) for soccer players. J Sports Med Phys Fit, 2014; 56(1-2): 34-38

Di Salvo V, Baron R, Tschan H, Calderon Montero FJ, Bachl N, Pigozzi F. Performance characteristics according to playing position in elite soccer. Int J Sports Med, 2007; 28(3): 222-227

Figueiredo AJ, Coelho-e-Silva MJ, Malina RM. Predictors of functional capacity and skill in youth soccer players. Scand J Med Sci Sports, 2011; 21: 446-454

Figueiredo AJ, Gonçalves CE, Coelho-e-Silva MJ, Malina RM. Youth soccer players, 11-14 years: maturity, size, function, skill and goal orientation. Ann Hum Biol, 2009; 36(1): 60-73

Freitas AS, Figueiredo AJ, de Freitas AL, Rodrigues VD, da Cunha AA, Deusdara FF, Coelho-e-Silva MJ. Biological Maturation, Body Morphology and Physical Performance in 8-16 year-old obese girls from Montes Claros - MG. J Hum Kinet, 2014; 43: 169-176

Harriss DJ, Atkinson G. International Journal of Sports Medicine - ethical standards in sport and exercise science research. Int J Sports Med, 2009; 30(10): 701-702

Hopkins W. Measures of reliability in sports medicine and science. Sports Med, 2000; 30: 1-15

Hopkins W. Sportscience 8, 2004. Available at: http:/www.sportsci.org/jour/04/wghtests.htm; accessed on 27.01.2015

Huijgen BC, Elferink-Gemser MT, Post W, Visscher C. Development of dribbling in talented youth soccer players aged 12-19 years: a longitudinal study. J Sport Sci, 2010; 28(7): 689-698

Jones PR, Pearson J. Anthropometric determination of leg fat and muscle plus bone volumes in young male and female adults. J Physiol, 1969; 204(2): 63-66

Khamis HJ, Roche AF. Predicting adult stature without using skeletal age: the Khamis-Roche method. Pediatrics, 1994; 94(4): 504-507

Lefevre J, Beunen G, Steens G, Claessens A, Renson R. Motor performance during adolescence and age thirty as related to age at peak height velocity. Ann Hum Biol, 1990; 17(5): 423-435

Lockie RG, Schultz AB, Callaghan SJ, Jeffriess MD, Berry SP. Reliability and Validity of a New Test of Change-of-Direction Speed for Field-Based Sports: the Change-of-Direction and Acceleration Test (CODAT). J Sports Sci Med, 2013; 12(1): 88-96

Malina RM, Cumming SP, Kontos AP, Eisenmann JC, Ribeiro B, Aroso J. Maturity-associated variation in sport-specific skills of youth soccer players aged 13-15 years. J Sport Sci, 2005; 23: 515-22

Malina RM, Eisenmann JC, Cumming SP, Ribeiro B, Aroso J. Maturity-associated variation in the growth and functional capacities of youth football (soccer) players 13-15 years. Eur J Appl Physiol, 2004; 91: 555-562

Malina RM, Pena Reyes ME, Eisenmann JC, Horta L, Rodrigues J. Miller R. Height, mass and skeletal 
maturity of elite Portuguese soccer players aged 11-16 years. J Sport Sci, 2000; 18: 685-693

Mendez-Villanueva A, Buchheit M, Kuitunen S, Douglas A, Peltola E, Bourdon P. Age-related differences in acceleration, maximum running speed, and repeated-sprint performance in young soccer players. $J$ Sport Sci, 2011; 29(5): 477-484

Meyers RW, Oliver JL, Hughes MG. Maximal Sprint Speed in Boys of Increasing Maturity. Pediatr Exerc Sci, 2015; 27: 85-94

Mueller WM, Martorell R. Reliability and accuracy of measurement. In: Lohman TG, Roche AF, Martorell R, eds. Anthropometric standardization reference manual. Champaign, IL: Human Kinetics, 83-86; 1988

Reilly T, Williams AM, Nevill A, Franks A. A multidisciplinary approach to talent identification in soccer. J Sport Sci, 2000; 18: 695-702

Robinson G, O'Donoghue P, Wooster B. Path changes in the movement of English Premier League soccer players. J Sport Med Phys Fit, 2011; 51: 220-226

Slaughter MH, Lohman TG, Boileau RA, Horswill CA, Stillman RJ, Van Loan MD, Bemben DA. Skinfold equations for estimation of body fatness in children and youth. Hum Biol, 1988; 60(5): 709-723

Spencer M, Pyne D, Santisteban J, Mujika I. Fitness determinants of repeated-sprint ability in highly trained youth football players. Int J Sports Physiol Perform, 2011; 6(4): 497-508

Tessitore A, Perroni F, Cortis C, Meeusen R, Lupo C, Capranica L. Coordination of soccer players during preseason training. J Strength Cond Res, 2011; 25: 3059-3069

Vaeyens R, Malina RM, Janssens M, Van Renterghem B, Bourgois J, Vrijens J, Philippaerts RM. A multidisciplinary selection model for youth soccer: the Ghent Youth Soccer Project. Br J Sports Med, 2006; 40: 928-934

Young W, Hawken M, McDonald L. Relationship between speed, agility and strength qualities in Australian Rules Football. Strength Cond Coach, 1996; 4: 3-6

\section{Corresponding author:}

\section{Manuel J Coelho-e-Silva}

Faculty of Sports Science and Physical Education, University of Coimbra, Portugal

Estádio Universitário de Coimbra, Pavilhão III

3040-156 Coimbra, Portugal

Phone number: +351 964809054

Fax number: +351 239802779

E-mail: mjcesilva@hotmail.com 